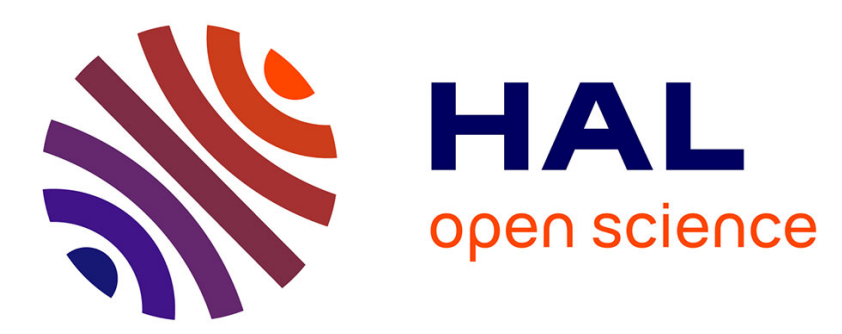

\title{
Design and characterization of bubble phononic crystals
} Valentin Leroy, Alice Bretagne, Mathias Fink, Herve Willaime, Patrick Tabeling, Arnaud Tourin

\section{To cite this version:}

Valentin Leroy, Alice Bretagne, Mathias Fink, Herve Willaime, Patrick Tabeling, et al.. Design and characterization of bubble phononic crystals. Applied Physics Letters, 2009, 95 (17), pp.1. 10.1063/1.3254243. hal-00515482

\section{HAL Id: hal-00515482 \\ https://hal.science/hal-00515482}

Submitted on 7 Sep 2010

HAL is a multi-disciplinary open access archive for the deposit and dissemination of scientific research documents, whether they are published or not. The documents may come from teaching and research institutions in France or abroad, or from public or private research centers.
L'archive ouverte pluridisciplinaire HAL, est destinée au dépôt et à la diffusion de documents scientifiques de niveau recherche, publiés ou non, émanant des établissements d'enseignement et de recherche français ou étrangers, des laboratoires publics ou privés. 


\title{
Design and characterization of bubble phononic crystals
}

\author{
Valentin Leroy, ${ }^{*}$ Alice Bretagne, Mathias Fink, and Arnaud Tourin \\ Institut Langevin, ESPCI ParisTech, CNRS UMR 7587, \\ Université Paris 7 Laboratoire Ondes et Acoustique, \\ 10 rue Vauquelin, 75231 Paris Cedex 05, France \\ Hervé Willaime and Patrick Tabeling \\ MMN, Gulliver, ESPCI ParisTech, 10 rue Vauquelin 75005 Paris, France
}

(Dated: October 5, 2009)

\begin{abstract}
We report the practical realization of phononic crystals with gas inclusions, using soft lithography techniques. Ultrasonic experiments from 0.3 to $5 \mathrm{MHz}$ confirm the existence of deep and wide minima of transmission through the crystal. We show that the first gap is due to the combined effects of Bragg reflections and bubble resonances. We propose a simple layered model that gives a reasonable prediction of the ultrasonic transmission.
\end{abstract}

Multiple scattering of waves is responsible for a wide range of fascinating phenomena such as band gaps in periodic structures $[1,2]$ and strong localization in disordered media [3]. Ultrasound in the $\mathrm{MHz}$ range are particularly well-suited to study these phenomena because in that case samples are macroscopic and the phase of the wavefield can be directly measured. Taking advantage of these two points, weak and strong localization have been studied with ultrasound in the past years $[4,5]$. As to strong localization, bubble clouds have been presented as promising candidates for observing it [6]. It has also been predicted that crystals made of air inclusions in a fluid matrix must exhibit the widest band gaps ever reported $[7,8]$. The particular status of bubbles in acoustics comes from their huge resonance, the so-called Minnaert resonance, at a frequency $\omega_{0}=\sqrt{3 \beta_{\text {gas }} / \rho} / R$, where $\beta_{\text {gas }}$ is the longitudinal modulus of the gas, $\rho$ the mass density of the liquid, and $R$ the radius of the bubble. This resonance has a special feature, responsible for the uncommon properties of bubbly media, which is its low frequency: $\omega_{0} R / v \ll 1$, where $v=\sqrt{\beta / \rho}$ is the velocity of sound in the liquid.

Nevertheless, no experimental evidence of localization through disordered bubble clouds or band gaps through bubble crystals have been brought until now. Two reasons are experimental: building a perfectly controllable sample with monodisperse bubble size is a difficult task. Another difficulty is the strong absorption occurring at resonance, which can make transmission results difficult to interpret. Besides experimental issues, some debates still exist concerning the theoretical interpretation of numerical works, although absorption and bubble size can be tuned as wanted in that case. Some authors have indeed reported a huge decrease in acoustic transmission through both periodic and disordered bubble assemblies near individual resonances of bubbles and have interpreted it as a manifestation of acoustic localization [9].

In this article, we propose a solution to tackle the experimental difficulties associated with the production and control of bubbles. The idea is to have bubbles em- bedded in an elastic matrix rather than a liquid. In that case, the shear modulus $\mu$ of the matrix plays a role in the resonance frequency which becomes $\omega_{0}=$ $\sqrt{\left(3 \beta_{\text {gas }}+4 \mu\right) / \rho} / R[10]$. Therefore, it appears that a bubble in a stiff medium $(\mu \simeq \beta)$ does not exhibit a low frequency resonance. However if the matrix is soft enough $(\mu \ll \beta)$, the low-frequency character of the resonance is preserved, implying that bubbly soft elastic materials would also have interesting acoustic properties, as bubbly liquids. The practical interests are significant: while bubbly liquids are unstable and difficult to control, bubbly soft elastic media can be steady and designed.

We present an example of such a bubbly soft elastic material: a phononic crystal made of polydimethylsiloxane (PDMS) with air inclusions. PDMS complies with the soft-medium requirement: $\mu \simeq 0.6 \mathrm{MPa}, \beta \simeq$ $1 \mathrm{GPa}$ [11]. Besides, its mechanical and optical properties have made it a first choice material for many microelectromechanical system (MEMS) applications, which means that well-established micro-fabrication techniques have been developped for PDMS.

The samples were fabricated in RTV615 (Bayers Silicones), using the standard soft lithography procedure employed in microfluidics.

We created $5 \mathrm{~cm}$-diameter molds with $2 \mathrm{D}$ periodic arrangements of cylinders (diameter $d=78 \mu \mathrm{m}$, heigth $h=50 \mu \mathrm{m}$, lattice constant $\left.a_{\mathrm{x}}=a_{\mathrm{y}}\right)$. A thin layer of PDMS (monomer and hardener with a 10:1 weight ratio) was then deposited on the mold by spincoating (50 seconds at $100 \mathrm{rpm}$ ) and cured at $70^{\circ} \mathrm{C}$ for 1 hour. By stacking $N$ patterned layers and closing the structure with a pure PDMS layer, we obtained a 3D crystal such as the one depicted in Fig. 1. Two different molds were fabricated with different lattice constants: $a_{\mathrm{x}}=300 \mu \mathrm{m}$ and $a_{\mathrm{x}}=200 \mu \mathrm{m}$. From these two molds, two 1-layer crystals and two 4-layer crystals with $a_{\mathrm{z}}=360 \mu \mathrm{m}$ were created.

Alignment of the layers was a problematic issue. The results shown in this article were obtained with imperfect crystals: each layer was perfectly ordered and successive 


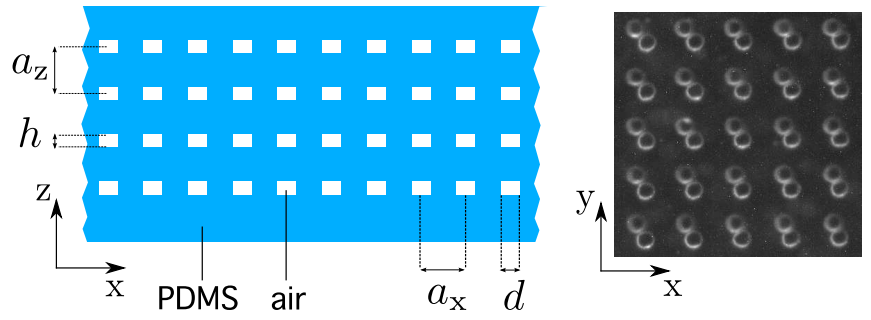

FIG. 1: (Left) Side-view schematic of a 4-layer phononic crystal. (Right) Top view of two successive misaligned layers.

layers were separated by the same distance, but alignment from one layer to the other was not insured (see Fig. 1). Ultrasonic measurements were carried out in a large water tank. A piezoelectric transducer generated a short pulse that propagated through water, traversed the sample along $z$ direction, and reached a receiving transducer. The sample was placed in the far field of the transducers and, to reduce spurious signals coming from the edges, it was mounted on the aperture ( $4 \mathrm{~cm}$-diameter) of an acoustically opaque screen. The transmission factor was obtained by calculating the ratio of the Fourier transforms of the signals acquired with and without the sample in the path of the acoustic beam. Two pairs of transducers were used, with central frequencies of 1 and $3.5 \mathrm{MHz}$, allowing us to measure the transmission between 0.3 and $5 \mathrm{MHz}$.

It was checked that there was no ultrasonic reflection from the interface between two layers of plain PDMS, thus insuring that our samples of stacked layers could be considered as 3D blocks of PDMS with air inclusions. We also measured the phase velocity and attenuation of ultrasound in pure PDMS and found $v=1.02 \mathrm{~mm} / \mu \mathrm{s}$, in accordance with previously reported values [12], and $\alpha=0.023(\omega / 2 \pi)^{1.45} \mathrm{~mm}^{-1}$ (with frequency $\omega / 2 \pi$ in $\mathrm{MHz}$ ). Figure 2 shows the measured ultrasonic transmission along the $z$ axis through a 4-layer crystal with $a_{\mathrm{x}}=300 \mu \mathrm{m}, a_{\mathrm{z}}=360 \mu \mathrm{m}$. There is a deep minimum in transmission at $0.6 \mathrm{MHz}$, and a less marked one at 1.6 MHz. Considering the finite thickness of the sample $(1.8 \mathrm{~mm})$, the depth of the first minimum is remarkably large. In comparison, the first gap for a 4-layer crystal of close-packed $0.8 \mathrm{~mm}$ diameter tungsten carbide beads in water is one order of magnitude less deep [13]. As shown in figure 3 , a bubble phononic crystal can offer even deeper band gaps when the number of bubbles per unit volume is increased: with $a_{\mathrm{x}}=200 \mu \mathrm{m}$, the two minima are more pronounced at 0.8 and $1.7 \mathrm{MHz}$, and there is a third small minimum around $3 \mathrm{MHz}$.

It is interesting to compare transmission through the 4-layer crystals and through their corresponding 1-layer crystals (circles in figures 2 and 3): even with a single layer, the first minimum is visible, at the same frequency. It has been shown that this minimum in transmission was due to the resonance of the air inclusions [14]. However, bubbles resonance does not alone explain the depth of the first minimum. Indeed, the horizontal dotted lines in figures 2 and 3 correspond to the fourth power of the minimum of transmission through the 1-layer crystals, i.e. the level one can expect through the 4-layer crystals if multiple reflections are neglected. The measured minima are 2 to 4 times deeper because the Bragg reflections also contribute to the decrease of the transmission in this frequency range: for $a_{\mathrm{z}}=360 \mu \mathrm{m}$, the first Bragg minimum is indeed expected at $0.7 \mathrm{MHz}$.

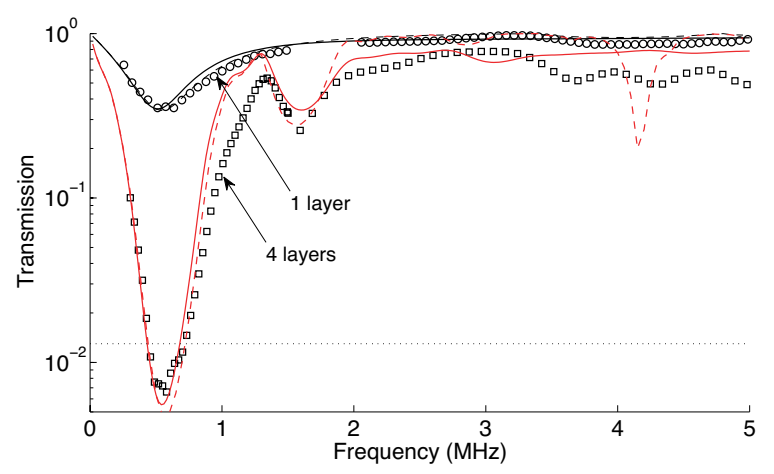

FIG. 2: Transmission of ultrasound measured through a 1layer crystal (circles) and a 4-layer crystal (squares) with lattice constants $a_{\mathrm{x}}=300 \mu \mathrm{m}$ and $a_{\mathrm{z}}=360 \mu \mathrm{m}$. Solid and dashed lines: predictions of the layered model and MST, respectively. Horizontal dotted line: minimum of the one-layer transmission to the fourth power.

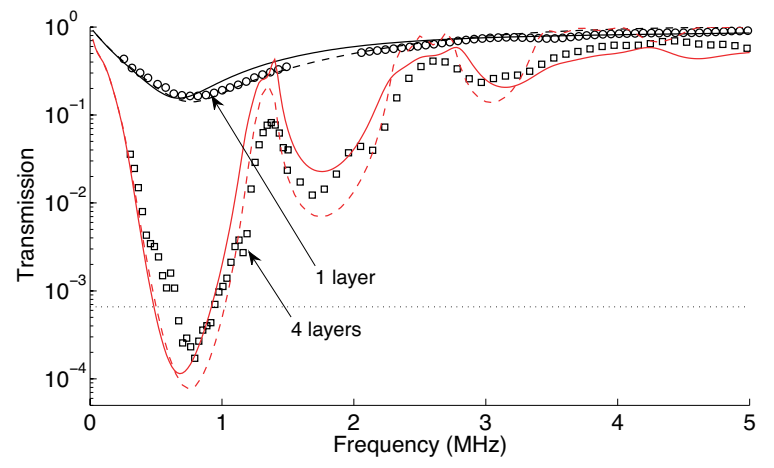

FIG. 3: Transmission of ultrasound measured through a 1layer crystal (circles) and a 4-layer crystal (squares) with lattice constants $a_{\mathrm{x}}=200 \mu \mathrm{m}$ and $a_{\mathrm{z}}=360 \mu \mathrm{m}$. Solid and dashed lines: predictions of the layered model and MST, respectively. Horizontal dotted line: minimum of the one-layer transmission to the fourth power.

We propose a simple model in which each layer of bubbles is treated as a partially reflective infinitely thin layer with a reflection coefficient $r$ and a transmission coefficient $t=1+r$. An iterative process then leads to the 
following expressions for the reflection $R_{N}$ and the transmission $T_{N}$ through $N$ thin layers $(N>1)$ separated by a distance $a_{\mathrm{z}}$ :

$$
\begin{aligned}
T_{N+1} & =\frac{t T_{N}}{1-R_{N} r \mathrm{e}^{2 \mathrm{i} k a_{\mathrm{z}}}} \\
R_{N+1} & =r+R_{N} \frac{t^{2} \mathrm{e}^{2 \mathrm{i} k a_{\mathrm{z}}}}{1-R_{N} r \mathrm{e}^{2 \mathrm{i} k a_{\mathrm{z}}}}
\end{aligned}
$$

where $k$ is the wave number in the material between the thin layers. For $N=1, T_{1}=t$ and $R_{1}=r$.

Following [14], we consider that the reflection from one layer of bubbles is given by

$$
r=\frac{K f}{1-K f \mathrm{e}^{\mathrm{i} k a_{\mathrm{x}} / \sqrt{\pi}}},
$$

where $K=2 \mathrm{i} \pi /\left(k a_{\mathrm{x}}^{2}\right)$, and $f$ the scattering function of the bubble: $f=R /\left(\omega_{0}^{2} / \omega^{2}-1-\mathrm{i} \delta\right)$, with $R$ the radius of the bubbles, $\omega_{0}$ their resonant angular frequency and $\delta$ their damping constant [15]. This expression was shown to be a good approximation for $k a_{\mathrm{x}} \ll 1$ and $a_{\mathrm{x}} / R>$ 3.6 [14]. Note that we assume that the wavelength is large enough compared to the size of the air inclusions to consider that cylinders are acoustically equivalent to spheres with $R=38 \mu \mathrm{m}$ (i.e., spheres with the same volume as the cylinders) [16].

The only unknown parameter in our layered model is the shear modulus of the PDMS $\mu=\mu^{\prime}+\mathrm{i} \mu^{\prime \prime}$. Good agreement is found when $\mu^{\prime}=\mu^{\prime \prime}=1.6 \mathrm{MPa}$ (see figures 2 and 3 ). This value seems realistic because it lies between the low frequency value $(0.6 \mathrm{MPa})$ and the value we found at $10 \mathrm{MHz}$ with shear waves velocity measurements $\left(\mu^{\prime}=\mu^{\prime \prime} \simeq 2.5 \mathrm{MPa}\right)$. We also computed a multiple scattering theory (MST) calculation with the same parameters, using the program MULTEL [17]. Both models give correct predictions of the positions and levels of the transmission minima. Agreement between the models is an indication that, in our samples and for normal incidence, band gaps are robust to bad alignment from one layer to the other. We have shown that phononic crystals of bubbles could be designed using soft lithography techniques. Transmission measurements in $\Gamma Z$ direction of the crytals, over the $0.3-5 \mathrm{MHz}$ frequency range, exhibited deep minima, which we interpreted as band gaps. We showed that, for the first band gap, the resonance of the bubbles conjugated with the Bragg reflections to block the propagation of acoustic waves. A simple model was proposed which gives good predictions of the transmission. As this model has an easy analytical expression, it can be useful for a quick estimation of the properties of a phononic crystals of bubbles. Although the technique we used to create crystals of bubbles is perfectible (in particular alignment), it offers promissing perspectives for the design of acoustic metamaterials using the low frequency resonance of bubbles to taylor new acoustic properties.

We thank R. Sainidou for providing an extended version of Multel, and F. Monti for helping us in the microfabrication process.

* Present address: Laboratoire Matière et Systèmes Complexes, Université Paris Diderot-Paris 7, CNRS (UMR 7057), France; Electronic address: valentin.leroy@ univ-paris-diderot.fr

[1] M. S. Kushwaha, P. Halevi, G. Martínez, L. Dobrzynski, and B. Djafari-Rouhani, Phys. Rev. B 49, 2313 (1994).

[2] A. Khelif, A. Choujaa, S. Benchabane, B. DjafariRouhani, and V. Laude, Applied Physics Letters 84, 4400 (2004).

[3] A. Lagendijk, B. van Tiggelen, and D. S. Wiersma, Physics Today 62, 24 (2009).

[4] A. Tourin, A. Derode, P. Roux, B. A. van Tiggelen, and M. Fink, Phys. Rev. Lett. 79, 3637 (1997).

[5] H. Hu, A. Strybulevych, J. H. Page, S. E. Skipetrov, and B. A. van Tiggelen, Nature Physics 4, 945 (2008).

[6] C. A. Condat and T. R. Kirkpatrick, Phys. Rev. B 36, 6782 (1987).

[7] M. S. Kushwaha, B. Djafari-Rouhani, and L. Dobrzynski, Physics Letters A 248, 252 (1998).

[8] M. Kafesaki, R. S. Penciu, and E. N. Economou, Phys. Rev. Lett. 84, 6050 (2000).

[9] Z. Ye and H. Hsu, Applied Physics Let. 79, 1724 (2001).

[10] V. N. Alekseev and S. A. Rybak, Acoustical Physics 45, 535 (1999).

[11] F. Schneider, T. Fellner, J. Wilde, and U. Wallrabe, J. Micromech. Microeng. 18, 1 (2008).

[12] C. G. Delides and T. A. King, J. Chem. Soc., Faraday Trans. 2 75, 359 (1979).

[13] J. H. Page, A. Sukhovich, S. Yang, M. L. Cowan, F. V. D. Biest, A. Tourin, M. Fink, Z. Liu, C. T. Chan, and P. Sheng, physica status solidi (b) 241, 3454 (2004).

[14] V. Leroy, A. Strybulevych, M. G. Scanlon, and J. H. Page, Eur. Phys. J. E 29, 123 (2009).

[15] T. Leighton, The Acoustic Bubble (Academic Press, 1994).

[16] M. Strasberg, J. Acoust. Soc. Am. 25, 536 (1953).

[17] R. Sainidou, N. Stefanou, I. Psarobas, and A. Modinos, Computer Physics Communications 166, 197 (2005). 